\title{
Steroids in leprosy type 1 (reversal) reactions: mechanisms of action and effectiveness
}

\author{
D. N. J. LOCKWOOD \\ Clinical Research Unit, Department of Infectious and Tropical \\ Diseases, London School of Hygiene \& Tropical Medicine, London, \\ UK
}

Summary Steroids are widely used for the treatment of leprosy reactions. The effectiveness of steroid treatment is variable, with only $60 \%$ of patients regaining nerve function. Sequential skin biopsy specimens, obtained from 15 patients with type 1 (reversal) reactions, have been studied to document the cytokine profile and cellularity of the lesions. All of the patients were placed on a standard course of steroids after the first biopsy. Subsequent biopsies were performed seven, 28 and 180 days later. The specimens were stained for interferon- $\gamma$ (IFN $\gamma$ ), interleukin-12 (IL12) and inducible nitric oxide synthase (iNOS). After the first biopsy, all patients were placed on a standard reducing course of steroids beginning at $30 \mathrm{mg}$ daily.

By day 7 , treatment with prednisolone showed little effect on the cellularity and cytokine profiles. However, by day 28 , significant decreases of IFN- $\gamma$, IL- 12 and iNOS were found for most patients. Some patients maintained cytokine production at day 28 and even at day 180 . These data illustrate the strong Thl profile of type 1 reactional lesions, the relatively slow response to therapy, and the continuing activity after treatment with steroids for 180 days. The variation of individual responses emphasizes their importance. Additional prospective studies will be required to determine whether patients with high intra-lesional levels of cytokine are at risk of recurrent reactions. The need for studies both of different glucocorticoids and of other non-steroidal immunosuppressants for the treatment of reactions is discussed.

\section{Introduction}

This paper focuses on the use of steroid treatment for leprosy reactions and neuritis, considering first the outcome of steroid treatment, and then the mechanisms by which steroids work at the molecular level. Some data from a study being carried out in Hyderabad, India, on the effects of steroid treatment on the cytokine profiles of reactional skin lesions are presented, and the implications of developing better treatment of reactions are discussed.

Corticosteroids are widely used in both hospital and field settings for the treatment of leprosy reactions and nerve damage. Semi-standardized, reducing courses of steroids, lasting for 12 weeks, are recommended by the World Health Organisation. ${ }^{1}$ However, the data with respect to the outcome of treatment with steroids show a range of outcomes. In an outpatient clinic in Hyderabad, India, only $50 \%$ of patients with reactions were judged to have improved nerve function after steroid treatment. ${ }^{2}$

Recently, data have become available from prospective field studies. In Bangladesh, full 
recovery was found in only $33 \%$ of motor and $37 \%$ of sensory nerves, whereas $31 \%$ of motor and $32 \%$ of sensory nerves failed to improve, and $12 \%$ of motor and $13 \%$ of sensory nerves deteriorated in the course of steroid treatment. ${ }^{3}$ The response to treatment is also affected by the length of time that the neuritis has been present. In an Ethiopian study, $88 \%$ of patients who developed acute neuritis after treatment, and whose neuritis was detected and treated promptly, made a full recovery. However, among the patients with chronic neuritis, only $51 \%$ of the affected nerves recovered fully. ${ }^{4}$

Steroids have both physicochemical and genomic effects. Their non-genomic effects are either receptor-mediated or mediated via non-specific physicochemical activity. ${ }^{5}$ These actions are probably responsible for the immediate actions of steroids, such as reducing oedema. The genomic actions of steroids involve the glucocorticoid crossing cell membranes, attaching to glucocorticoid receptors, and binding to glucocorticoid response elements on the genomic DNA. It is this mechanism that affects cytokine production. ${ }^{6}$ Multiple effects of steroids on cytokine production have been described, with actions proposed at the levels of transcription and translation, and in the stability of mRNA and protein products. ${ }^{7}$ The principal mechanism is thought to be inhibition of NF- $\kappa$ B-induced transcription of cytokinemRNAs. A number of cytokine genes have the same NF- $\kappa \mathrm{B}$ binding sites in their promoter regions, and the production of several pro-inflammatory cytokines, including tumour necrosis factor- $\alpha$ (TNF $\alpha)^{8}$ interleukin-2 (IL-2), ${ }^{9}$ IL- $12^{10}$ and interferon- $\gamma(\text { IFN } \gamma)^{11}$ are downregulated by glucocorticoids. The same mechanism has been shown to be involved in the down-regulation of expression of inducible nitric oxide synthase (iNOS) by corticosteroids. ${ }^{12}$ Increased degradation of iNOS has also been noted as an effect of steroids.

There have been few examinations of the effect of steroids on local cytokine production within inflammatory lesions in vivo. In this present study, skin lesions of leprosy patients with type 1 reactions were sampled sequentially to determine the effects of treatment with prednisolone on local cell population and cytokine profiles. Another aim was to determine whether steroids switch off the Th1 response associated with type 1 reactions. The detailed methods and results of this study are being reported elsewhere. ${ }^{13}$

\section{Methods and results}

Biopsies were performed on days 0, 7, 28 and 180 from 15 (six BT and nine BL) patients with clinically diagnosed and histologically confirmed type 1 reactions. After the first biopsy, all patients were placed on a standard reducing course of steroids, starting at $30 \mathrm{mg}$ daily. Staining for IFN $\gamma$, IL-12 and iNOS proteins and CD3, CD4 and CD8 cell types, was performed on cryosections, employing monoclonal antibodies in a two-stage immunoperoxidase technique. ${ }^{14}$ Positive staining was visualized using 3,3-diaminobenzidine. The location and amount of staining for each cell and cytokine at each time-point was assessed.

In the day 0 biopsy specimen, all patients demonstrated granulomata with strongly positive staining for IFN $\gamma$, IL-12 and iNOS. Treatment with prednisolone had little effect on the cellularity and cytokine profiles at day 7. By day 28 , however, significant decreases were found for the cellularity of the lesions and for production of IFN $\gamma$, IL-12 and iNOS in most but not all patients. No correlations were found between clinical severity of disease type (BT or BL) and cytokine production. Only five patients were biopsied at 180 days, all of them BL patients. Three of these patients still had very active lesions, all with increased cytokine levels compared to the levels at 28 days. 


\section{Discussion}

This study illustrates the strong Th 1-like cytokine-profile within reactional lesions. Although prednisolone decreases the production of IFN $\gamma$, IL-12 and iNOS, it does so slowly. This is rather surprising, given the rapid action of glucocorticoids (even the genomic actions require only a few hours). ${ }^{15}$ Furthermore, many BL patients still have considerable activity in their lesions after 6 months. It will be important to identify the factors that drive the ongoing recruitment of cells into the lesions. These data also illustrate the need to study lesions within individual patients over a long time-span.

What are the implications of these observations for the development of medical therapies for reactions? Damage to nerves might be reduced if drugs with a more rapid effect on the cellularity and lesional cytokine profile were used for treatment. Recent work on the relative genomic and non-genomic potencies of glucocorticoids suggests that glucocorticoids with a high ratio of non-genomic to genomic activity may be needed for early, high-level immunosuppression. ${ }^{15}$ It may be that methylprednisolone, which has such a ratio, is a better drug for initiating treatment.

In BL patients, there may be a need for prolonged immunosuppression. It is interesting that, in the Ethiopian study, patients with chronic neuritis had only a $51 \%$ improvement of nerve function; it may be that these patients represent a subgroup characterized by ongoing immune mediated activity in their lesions. Further prospective studies are needed to determine whether patients with high or prolonged intralesional cytokine production are at risk of recurrent reactions.

There has been no formal experience in leprosy of using non-steroidal immunosuppressants to treat reactions. As part of the INFIR collaboration, studies of two such immunosuppressants, used extensively in the treatment of other immune-mediated disease processes, are beginning. In these studies, cyclosporin A and azathioprine will be compared to prednisolone in the management of reactions. Cyclosporin A inhibits transcription of IL-2 mRNA, thereby blocking proliferation of T cells, whereas azathioprine inhibits synthesis of nucleic acids, and acts much more slowly. Combinations of immunosuppressants have been used with success in other immune-mediated conditions such as rheumatoid arthritis. ${ }^{16}$ The experience gained with other agents should be examined so that, where appropriate, the new agents may be subjected to clinical trial in leprosy reactions and neuritis.

It may be that the ideal immunosuppressant for leprosy reactions will be a combination of therapies - a drug with a fast-acting component that will switch inflammation off more rapidly than does prednisolone, and a longer-acting immunosuppressant that will ameliorate ongoing inflammation in patients who are at risk of chronic inflammation. It is important that clinically based studies of molecular predictors of outcomes in leprosy reactions be carried out, and that appropriate new drugs be tried for the large numbers of patients who do not respond to steroids.

\section{Acknowledgements}

I wish to thank Drs Saroj Young, Sujai Suneetha, David Little and Anne Coulthart, all of whom contributed to the experimental work discussed here. The patient-studies were done as a collaboration between The London School of Hygiene \& Tropical Medicine and Dhoolpet Leprosy Research Centre (DLRC), Hyderabad, India. DLRC is supported by the Medical 
Research Council (UK) through LEPRA-India. This work is supported by Glaxo-Wellcome through LEPRA.

\section{References}

1 WHO Expert Committee on Leprosy. WHO Technical Report Series no. 874. World Health Organization, Geneva, 1998.

2 Lockwood DNJ, Vinayakumar S, Stanley JNA et al. The clinical features and outcome of reversal (type 1) reactions in Hyderabad, India. Int J Lepr, 1993; 61: 8-15.

3 Croft PR, Nicholls PG, Richardus JH, Smith WCS. The treatment of acute nerve function impairment in leprosy: results from a prospective cohort study in Bangladesh. Lepr Rev, 2000; 71: 154-168.

${ }^{4}$ Saunderson P, Gibre S, Desta K et al. The pattern of neuritis in the AMFES patients: definitions, incidence, risk factors and outcome. Lepr Rev, 2000; in press.

5 Buttgereit F, Wehling M, Burmester GR. A new hypothesis of modular glucocorticoid actions. Glucocorticoid treatment of rheumatic diseases revisited. Arthr Rheum, 1998; 41: 761-767.

${ }^{6}$ Bames PJ. Anti-inflammatory actions of glucocorticoids: molecular mechanisms. Clin Sci, 1998; 94: 557-572.

7 Barnes PJ, Adcock I. Anti-inflammatory actions of steroids: molecular mechanisms. Trends Pharmacol Sci, 1993; 14: 436-441.

${ }^{8}$ Han J, Thompson P, Beutler B. Dexamethasone and pentoxyfylline inhibit endotoxin-induced cachectin/tumour necrosis factor synthesis at separate points in the signaling pathway. J Exp Med, 1990; 172: 391-394.

9 Paliogianni F, Raptis A, Ahuja SS et al. Negative transcriptional regulation of human interleukin 2 (IL-2) gene by glucocorticoids through interference with nuclear transcription factors AP-1 and NF-AT. J Clin Invest, 1993; 91: 1481-1489.

10 DeKruyff RH, Fang Y, Umetsu DT. Corticosteroids enhance the capacity of macrophages to induce Th2 cytokine synthesis in CD4+ lymphocytes by inhibiting IL-12 production. J Immunol, 1998; 160: 2231-2237.

11 Arya SK, Wong-Staal F, Gallo RC. Dexamethasone-mediated inhibition of human T cell growth factor and $\gamma$-interferon messenger RNA. J Immunol, 1984; 133: 273-276.

12 Walker G, Pfeilschifter J, Kunz D. Mechanisms of suppression of inducible nitric-oxide synthase (iNOS) expression in interferon (IFN)-gamma-stimulated RAW 264.7 cells by dexamethasone. Evidence for glucocorticoid-induced degradation of iNOS protein by calpain as a key step in post-transcriptional regulation. J Biol Chem, 1997; 272: 16679-16687.

13 Little D, Khanolkar-Young S, Coulthart A et al. Immunohistochemical analysis of cellular infiltrate, IFN $\gamma$, IL-12 and iNOS expression in leprosy type 1 (reversal) reactions before and during prednisolone treatment. Infect Immun, submitted.

14 Khanolkar-Young S, Rayment P, Brickell PM et al. Tumour necrosis factor- $\alpha$ synthesis is associated with the skin and peripheral nerve pathology of leprosy reversal reactions. Clin Exp Immunol, 1995; 99: 196-201.

15 Lipworth BJ. Therapeutic implications of non-genomic glucocorticoid activity. Lancet, 2000; 356: 87-88.

${ }^{16}$ Furst DE. Aggressive strategies for treating aggressive rheumatoid arthritis: has the case been proven? Lancet, 2000; 356: $183-184$.

\section{DISCUSSION}

Dr Sampaio: We carried out a similar study, in which we biopsied the lesions of patients in reaction and carried out PCR on the lesions. Steroid therapy brings about a decrease of expression of TNF $\alpha$ mRNA, but an increase of expression of IL-10 mRNA. In the case of patients with ENL who are treated with thalidomide, one does not see the increase of expression of IL-10 mRNA. 